

Published in final edited form as:

Nature. 2009 September 3; 461(7260): 95-98. doi:10.1038/nature08324.

\title{
Reptilian heart development and the molecular basis of cardiac chamber evolution
}

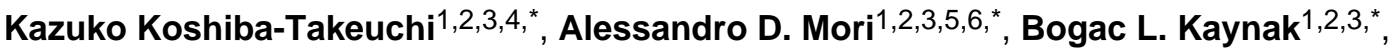
Judith Cebra-Thomas ${ }^{7}$, Tatyana Sukonnik ${ }^{1,2,3}$, Romain O. Georges ${ }^{8}$, Stephany Latham ${ }^{9}$, Laural Beck $^{9}$, R. Mark Henkelman ${ }^{10,11}$, Brian L. Black ${ }^{3,12}$, Eric N. Olson ${ }^{13}$, Juli Wade ${ }^{9}$, Jun K. Takeuchi ${ }^{4}$, Mona Nemer ${ }^{8,14}$, Scott F. Gilbert ${ }^{15}$, and Benoit G. Bruneau ${ }^{1,2,3,5,6}$

${ }^{1}$ Gladstone Institute of Cardiovascular Disease, San Francisco, California 94158, USA

${ }^{2}$ Department of Pediatrics, University of California, San Francisco, California 94158, USA

${ }^{3}$ Cardiovascular Research Institute, University of California, San Francisco, California 94158, USA

${ }^{4}$ Division of Cardiovascular Research, Global-Edge Institute, Tokyo Institute of Technology,

Ontario M5G 1X8, Canada

${ }^{6}$ Department of Molecular Genetics, University of Toronto, Toronto, Ontario M5S 1A8, Canada

${ }^{7}$ Biology Department, Millersville University, Millersville, Pennsylvania 17551, USA

${ }^{8}$ Institut de Recherches Cliniques de Montréal, Programme de Biologie Moléculaire, Université de ontreal, Montreal, Québec H3C 3J7, Canada

Department of

\footnotetext{
Users may view, print, copy, download and text and data- mine the content in such documents, for the purposes of academic research, subject always to the full Conditions of use: http://www.nature.com/authors/editorial_policies/license.html\#terms

Author Information. Correspondence and requests for materials should be addressed to B.G.B. (bbruneau@ gladstone.ucsf.edu). These authors contributed equally to this work

Author Contributions. K.K-T. performed reptile histology and gene expression studies; A.D.M., B.K., T.S., and B.G.B. performed mouse experiments; J.C.-T. and S.F.G. obtained turtle specimens and isolated T. scripta Tbx5 cDNA; S.L. and L.B. isolated Anolis specimens under direction of J.W.; B.K. acquired and reconstructed OPT images; R.O.G. performed Tbx5 immunohistochemistry under direction of M.N.; R.M.H. directed initial mouse embryo OPT; J.K.T. obtained chick and mouse specimens; R.O.G., M.N., B.L.B., and E.N.O. provided genetically modified mice prior to publication; B.G.B. conceived and directed the project, and wrote the paper. All authors contributed to the written manuscript.

Supplementary Information accompanies the paper on www.nature.com/nature.

The authors declare no competing financial interests.
} 
${ }^{13}$ Department of Molecular Biology, University of Texas Southwestern Medical Center, Dallas, Texas 75390, USA

${ }^{14}$ Department of Biochemistry, Microbiology and Immunology, University of Ottawa, Ottawa, Ontario K1H 8M5 Canada

${ }^{15}$ Department of Biology, Swarthmore College, Swarthmore, Pennsylvania 19081, USA

\section{Abstract}

The emergence of terrestrial life witnessed the need for more sophisticated circulatory systems. This has evolved in birds, mammals, and crocodilians into complete septation of the heart into left and right sides, allowing separate pulmonary and systemic circulatory systems, a key requirement for the evolution of endothermy $1-3$. However, the evolution of the amniote heart is poorly understood. Reptilian hearts have been the subject of debate in the context of the evolution of cardiac septation: do they possess a single ventricular chamber or two incompletely septated ventricles4-7? We examined heart development in the red-eared slider turtle, Trachemys scripta elegans (a chelonian), and the green anole, Anolis carolinensis (a squamate), focusing on gene expression in the developing ventricles. Both reptiles initially form a ventricular chamber that homogenously expresses the T-box transcription factor gene Tbx5. In contrast, in birds and mammals, $T b x 5$ is restricted to left ventricle precursors8,9. In later stages, $T b x 5$ expression in the turtle (but not anole) heart is gradually restricted to a distinct left ventricle, forming a left-right gradient. This suggests that $T b x 5$ expression was refined during evolution to pattern the ventricles. In support of this hypothesis, we show that loss of $T b x 5$ in the mouse ventricle results in a single chamber lacking distinct identity, indicating a requirement for $T b x 5$ in septation. Importantly, misexpression of $T b x 5$ throughout the developing myocardium to mimic the reptilian expression pattern also results in a single mispatterned ventricular chamber lacking septation. Thus, ventricular septation is established by a steep and correctly positioned $T b x 5$ gradient. Our findings provide a molecular mechanism for the evolution of the amniote ventricle, and support the concept that altered expression of developmental regulators is a key mechanism of vertebrate evolution.

Amphibians have a three-chambered heart, while mammalian, crocodilian, and avian hearts have four chambers, two each for pulmonary and systemic circulations. The acquisition of a fully septated ventricle has evolved independently in birds, mammals, and crocodilians10, and is an important example of convergent evolution. Non-crocodilian reptiles (squamates, chelonians, and rhynchocephalians) hold a unique place in the evolution of the heart, as their ventricular chambers are apparent intermediates between these forms4-7. In reptiles, shunting can produce functional separations between left and right circulatory systems, but only complete septation allows a dual pressure system required for endothermy. Therefore, the evolutionary status of the reptilian ventricles is controversial7. Is it a primitive arrangement presaging the septated heart of crocodilians, birds, and mammals? Or is it an adaptation to particular circulatory requirements? Development of reptilian hearts has not been addressed in over 100 years 11, and thus the developmental basis of reptilian heart formation is not known. Furthermore, clear insight into the evolution of cardiac septation has not emerged from molecular studies of heart development3. 
Transcription factors of the T-box family are important regulators of heart formation12. One T-box gene, $T b x 5$, has an expression pattern that suggests a role in the evolution of cardiac septation (see Supplementary note 1). In amphibians, $T b x 5$ is expressed throughout the developing heart13. In birds and mammals, there is a steep gradient of $T b x 5$ expression from high levels in the prospective left ventricle (LV) to low levels in the prospective right ventricle (RV)8,9. Reduced dosage of Tbx5 in humans and mice leads to defects in interventricular septum (IVS) formation and patterning14-17, suggesting that a steep gradient of Tbx5 is critical for IVS formation. The evolutionary role of Tbx5 in septation is unknown.

We examined cardiac embryology of the red-eared slider turtle, $T$. scripta elegans (a chelonian), and the green anole, A. carolinensis (a squamate), focusing on the ventricles. Although the phylogenetic relationship of turtles to other reptiles is controversial based on anatomical considerations 18,19 , molecular phylogenies consistently group turtles with the archosaurs (birds and crocodiles)20,21. Anoles are considered to be more basal than archosaurs19-21. The post-hatching anole heart has a thick muscular ridge (Fig. 1a-d and Supplementary Fig. 1) that separates a proximal outflow tract, or cavum pulmonale6,11, from the main ventricular chamber. Turtles have a smaller muscular ridge and are thought to have a primitive IVS-like structure $4,6,11$, as we determined by three-dimensional reconstructions revealing a dense coalescence of trabeculae spanning the full depth of the heart (Fig. 1e-h and Supplementary Fig. 1). Initially, developing turtle and anole hearts showed no clear evidence of ventricular septation (Fig. 1i, and Supplementary Figs. 2-4). In contrast, the chick has a well-developed IVS at comparable early stages (Figs. 1i and Supplementary Fig. 3). In the turtle, a structure resembling an IVS appears only at stage 21 (Fig. 1i). Alligator embryos (Fig. 1j) have a muscular ridge and a distinct IVS. The muscular ridge has been interpreted as analogous to the IVS, leading to the impression that reptiles have multiple septa4-6. We speculate that the development of the muscular ridge in reptiles reflects persistent growth of the proximal outflow tract11, as seen transiently in chick heart (Fig. 1i-k and Supplementary Fig. 3).

To observe molecular patterning of reptile ventricles, we examined expression of Tbx5. In mammals and birds, $T b x 5$ mRNA and protein are highly enriched in the prospective LV (Fig. 2b,c and Supplementary Fig. 5)8,9. At looping heart tube stages, Tbx5 was broadly expressed throughout the embryonic turtle and anole hearts (Fig. 2a,d), similar to Xenopus Tbx5 (Ref 13), but unlike its early restricted expression in chick and mouse (Fig. 2b,c). In the anole, $T b x 5$ expression extended to the boundary of the ventricle and outflow tract, where the muscular ridge forms. At later stages, Tbx5 expression in turtle (stage 15) and anole (stage 13) remained homogeneous throughout the ventricle (Fig. 2e,h and data not shown). In comparable stages in chick, it was sharply restricted to LV primordium. At stages 17-18 in the turtle, Tbx5 mRNA levels decreased in RV primordium, remaining enriched in LV primordium, creating a steep left-right gradient, although not as sharply defined as in chick (Fig. 2f,g,h,i and Supplementary Figs. 6,7). This gradient was maintained at stage 21 (Fig. 2h,i). Tbx5 expression in Anolis was not restricted in the ventricle (Fig. 2f,i and Supplementary Fig. 7). We examined expression of Tbx 5 target genes expressed in trabeculae but excluded from mammalian IVS myocardium14,16,17,22. Bmp10 was 
expressed throughout the early turtle and anole trabeculae, but was excluded in turtles at Stage 17-18 from an expansion of the compact myocardium corresponding to presumptive IVS precursors, correlating with the boundary of $T b x 5$ expression (Fig. $2 \mathrm{j}$ and Supplementary Fig 6). This suggests a conserved molecular transition in the trabeculae that form the IVS. Turtle Nppa (not found in anoles23), formed a gradient similar to Tbx5 (Fig. $2 \mathrm{k})$. Thus, turtle ventricles, but not those of Anolis, acquire distinctions between left and right components late in development.

A steep Tbx 5 gradient in chick and mouse may have evolved to pattern the ventricles. Reducing Tbx5 levels supports this14,16,17. To address a potential role for Tbx5 in septation, we deleted $T b x 5$ from segments of developing mouse ventricles, using a conditionally deletable $T b x 5$ allele $\left(T b x 5^{\mathrm{LDN}}\right) 16$, and ventricular myocyte-specific $N k x 2.5:: C r e$ mice24 (Fig. 3a). These mice $\left(N k x 2.5: \because C r e^{t g / 0} ; T b x 5^{\mathrm{LDN} / \mathrm{LDN}}\right.$ mice, or $T b x 5^{\mathrm{V}-\mathrm{del}}$ mice) lacked morphological distinctions between the LV and RV that were obvious in wildtype embryos by embryonic day (E) 9.5 (Fig. 3b). Embryos with this univentricular phenotype persisted until E11.5 (Fig. 3c). Expression of Nppa and Bmp10, normally excluded from the interventricular groove, was expanded throughout the single ventricle of $T b x 5^{\mathrm{V} \text {-del }}$ embryos (Fig. 3e,f). Handl was expressed at lower levels, but in its normal domains, the LV and RV primordia (Fig. 3g). Thus, loss of Tbx5 from developing ventricles results in a single mispatterned ventricle.

To determine if a steep Tbx 5 gradient at the interventricular midpoint is critical for IVS formation, we deleted Tbx5 with Mef2cAHF::Cre mice25 (Fig. 3h). Since Mef2cAHF::Cre is active in RV and IVS precursors, but not in the LV free wall, the Tbx5 expression boundary is shifted leftward. Tbx $5^{\mathrm{LDN} / \mathrm{LDN}} ; \operatorname{Mef} 2 c A H F:: C r e\left(T b x 5^{\mathrm{AHF}-\mathrm{del}}\right)$ mice lacked an IVS (Fig. 3i-k). Gene expression analysis showed that a distinction between LV and RV was maintained (Fig. 31,m), but a clear absence of IVS-enriched markers (Irx2, Dkk3) at the ventricular midpoint, while maintained in the adjacent trabeculae, emphasize the absence of ventricular septation (Fig. 3n, Supplementary Fig. 8). Thus, a boundary of cells expressing high Tbx5 levels is necessary within a segment of myocardium where IVS outgrowth will occur. This implies a prepattern within which Tbx 5 must function; the nature of this prepattern is unknown (See supplementary note 2). Tbx5 expression and additional patterning cues may have co-evolved, or the prepattern may exist in all amniotes. Regardless, IVS formation requires a sharp Tbx 5 boundary indicating that $T b x 5$ patterning was a major factor in evolution of septation.

Our loss-of-function experiments demonstrate a requirement for $T b x 5$ in IVS formation distinct from a more global role in differentiation. These results do not address the evolutionary role of Tbx5 patterning; in particular, whether the broad expression of $T b x 5$ observed in anole and turtle would preclude IVS formation. Previous misexpression attempts yielded variable results ranging from no effect to severely malformed hearts (Ref. 9 and J.K.T., unpublished data). We misexpressed Tbx5 in the ventricles by crossing a mouse line bearing a stable Cre-activatable transgene expressing moderate Tbx5 levels upon induction (CAT-Tbx5)26 with Mef2cAHF::Cre or Nkx2.5::Cre mice (Fig. 4). CATTbx5; Mef2cAHF::Cre embryos survived until E11 and had a single ventricle at E10.25. Molecular analysis revealed expanded expression of Tbx5, Nppa, and Bmp10 across the interventricular 
groove of CAT-Tbx5;Mef2cAHF::Cre embryos (Fig. 4b). CAT-Tbx5;Nkx2.5::Cre embryos survived longer (until E12), presumably because this manipulation avoided secondary effects of Tbx 5 overexpression in cardiac progenitors. CAT-Tbx5;Nkx2.5::Cre embryos at E11.5 also had defective ventricular septation and mispatterned gene expression (Fig. 4c and

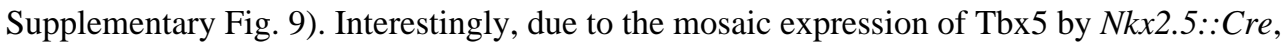
some embryos had no septum at all, while others with a more graded expression of Tbx 5 had a rudiment of a septum in which not all genes were mispatterned (Fig. $4 \mathrm{c}$ and Supplementary Fig. 9). Thus, misexpression of Tbx5 in a pattern reminiscent of the reptilian heart leads to loss of IVS patterning and morphogenesis, further supporting a role for $T b \times 5$ patterning in the evolution of septation.

Our results provide evidence that the reptilian heart, although evolved to function physiologically under conditions particular to reptilian life 7, is an evolutionary intermediate between amphibian and avian/crocodilian hearts in its ventricular development. The dynamic expression of $T b x 5$ and its leftward restriction suggest a temporal refinement model in which early restriction of $T b x 5$ expression to $\mathrm{LV}$ precursors, as seen in chick and mouse, provides a robust patterning cue for ventricular septation. In this model (Fig. 4d), a quantitative gradient of Tbx5 is essential for proper formation and patterning of the IVS. Our mouse genetic analyses, including decreased dosage14,16, are consistent with an important role for a steep gradient of Tbx 5 in chamber patterning and IVS formation. In the reptilian heart, the delayed and less pronounced establishment of this patterning may contribute to varying degrees of septation. Therefore patterning of $T b x 5$, in the archosaurian and synapsid lineages, is likely to be an important mechanism in the convergent evolution of septation. Our findings generally support the concept that altered expression of developmental regulators is an important aspect of morphological evolution27.

\section{METHODS SUMMARY}

Embryos were isolated from T. scripta elegans eggs (Kliebert Turtle and Alligator Farm, Hammond, LA). Green anole (A. carolinensis) embryos were collected in captivity. Mouse strains were described16,24-26. Whole-mount and section in situ hybridizations were performed using standard protocols. Immunohistochemistry and OPT were performed as previously described26,28. For all mouse experiments, at least 3 embryos were examined for each genotype at each stage, all with comparable results.

\section{Supplementary Material}

Refer to Web version on PubMed Central for supplementary material.

\section{Acknowledgements}

We thank J.N. Wylie and L. Davidson for technical assistance, M. Harris and J. Fallon for alligator embryos, T. Sanger and J. Gibson-Brown for unpublished data on Anolis staging, T. Ogura for chick Tbx5 and Tbx2O probes, and G. Howard and S. Ordway for editorial assistance. This work was funded in part by the March of Dimes Birth Defects Foundation (B.G.B.), the J. David Gladstone Institutes (B.G.B.), William H. Younger, Jr. (B.G.B.); an NIH PPG (P01HL089707, to B.G.B., B.L.B.); scholarships from the Natural Sciences and Engineering Research Council of Canada, the Heart and Stroke Richard Lewar Centre for Excellence, University of Toronto, and Ontario Graduate Scholarship (A.D.M.); the Fumi Yamamura Memorial Foundation for Female Natural Scientists and Grants-in-Aid for Scientific Research (C) (K.K-T), MEXT's program for young independent researchers (K.K-T, J.K.T.), 
Sumitomo Foundation and Nakajima Foundation (J.K.T), a Canada Research Chair in Imaging (R.M.H.), the Heart and Stroke foundation of Canada and the Canadian Institutes for Health Research (M.N.), and grants from the NSF (S.F.G. and J.C.-T., and J.W.). Funding for the J. David Gladstone Institutes from an NIH/NCRR grant (C06 RR018928) is also acknowledged.

\section{REFERENCES}

1. Farmer CG. Evolution of the vertebrate cardio-pulmonary system. Annu Rev Physiol. 1999; 61:573-592. [PubMed: 10099702]

2. Hillenius WJ, Ruben JA. The evolution of endothermy in terrestrial vertebrates: Who? When? Why? Physiol Biochem Zool. 2004; 77:1019-1042. [PubMed: 15674773]

3. Olson EN. Gene regulatory networks in the evolution and development of the heart. Science. 2006; 313:1922-1927. [PubMed: 17008524]

4. Holmes EB. A reconsideration of the phylogeny of the tetrapod heart. J Morph. 1975; 147:209-228.

5. Webb GJW. Comparative Cardiac Anatomy of the Reptilia. III. The heart of crocodilians and an hypothesis on the completion of the interventricular septum of crocodilians and birds. J Morph. 1979; 161:221-240.

6. Farrell, AP.; Gamperl, AK.; Francis, ETB. Biology of the Reptilia. Gans, C.; Gaunt, AS., editors. Vol. Vol. 19. Ithaca, NY: Soc. fort he Study of Amphibians and Reptiles; 1998. p. 375-424. (Morphology G)

7. Hicks JW. The physiological and evolutionary significance of cardiovascular shunting patterns in reptiles. News Physiol Sci. 2002; 17:241-245. [PubMed: 12433978]

8. Bruneau BG, Logan M, Davis N, et al. Chamber-specific cardiac expression of Tbx5 and heart defects in Holt- Oram syndrome. Dev Biol. 1999; 211:100-108. [PubMed: 10373308]

9. Takeuchi JK, Ohgi M, Koshiba-Takeuchi K, et al. Tbx5 specifies the left/right ventricles and ventricular septum position during cardiogenesis. Development. 2003; 130:5953-5964. [PubMed: 14573514]

10. Seymour RS, Bennett-Stamper CL, Johnston SD, et al. Evidence for endothermic ancestors of crocodiles at the stem of archosaur evolution. Physiol Biochem Zool. 2004; 77:1051-1067. [PubMed: 15674775]

11. Greil A. Beitrage zur vergleichenden Anatomie und Entwicklungsgeschichte des Herzens und des Truncus arteriosus der Wirbeltiere. Morphol. Jahrb. 1903; 31:123-310.

12. Stennard FA, Harvey RP. T-box transcription factors and their roles in regulatory hierarchies in the developing heart. Development. 2005; 132:4897-4910. [PubMed: 16258075]

13. Horb ME, Thomsen GH. Tbx5 is essential for heart development. Development. 1999; 126:1739_ 1751. [PubMed: 10079235]

14. Bruneau BG, Nemer G, Schmitt JP, et al. A murine model of Holt-Oram syndrome defines roles of the T-box transcription factor Tbx5 in cardiogenesis and disease. Cell. 2001; 106:709-721. [PubMed: 11572777]

15. Bruneau BG. The developmental genetics of congenital heart disease. Nature. 2008; 451:943-948. [PubMed: 18288184]

16. Mori AD, Zhu Y, Vahora I, et al. Tbx5-dependent rheostatic control of cardiac gene expression and morphogenesis. Dev Biol. 2006; 297:566-586. [PubMed: 16870172]

17. Koshiba-Takeuchi K, Takeuchi JK, Arruda EP, et al. Cooperative and antagonistic interactions between Sall4 and Tbx5 pattern the mouse limb and heart. Nat Genet. 2006; 38:175-183. [PubMed: 16380715]

18. Rieppel O. Turtle origins. Science. 1999; 283:945-946. [PubMed: 10075558]

19. Lyson T, Gilbert SF. Turtles all the way down: Loggerheads at the root of the chelonian tree. Evolution and Development. 2009; 11:133-135. [PubMed: 19245543]

20. Hedges SB, Poling LL. A molecular phylogeny of reptiles. Science. 1999; 283:998-1001. [PubMed: 9974396]

21. Shedlock AM, Botka CW, Zhao S, et al. Phylogenomics of nonavian reptiles and the structure of the ancestral amniote genome. Proc Natl Acad Sci U S A. 2007; 104:2767-2772. [PubMed: 17307883] 
22. Chen H, Shi S, Acosta L, et al. BMP10 is essential for maintaining cardiac growth during murine cardiogenesis. Development. 2004; 131:2219-2231. [PubMed: 15073151]

23. Trajanovska S, Donald JA. Molecular cloning of natriuretic peptides from the heart of reptiles: loss of ANP in diapsid reptiles and birds. General and comparative endocrinology. 2008; 156:339-346. [PubMed: 18295764]

24. McFadden DG, Barbosa AC, Richardson JA, et al. The Hand1 and Hand 2 transcription factors regulate expansion of the embryonic cardiac ventricles in a gene dosage-dependent manner. Development. 2004; 132:189-201. [PubMed: 15576406]

25. Verzi MP, McCulley DJ, De Val S, et al. The right ventricle, outflow tract, and ventricular septum comprise a restricted expression domain within the secondary/anterior heart field. Dev Biol. 2005; 287:437-449.

26. Georges R, Nemer G, Morin M, et al. Distinct expression and function of alternatively spliced Tbx5 isoforms in cell growth and differentiation. Mol Cell Biol. 2008; 28:4052-4067. [PubMed: 18391012]

27. Carroll SB. Evo-devo and an expanding evolutionary synthesis: a genetic theory of morphological evolution. Cell. 2008; 134:25-36. [PubMed: 18614008]

28. Lickert H, Takeuchi JK, von Both I, et al. Baf60c is essential for function of BAF chromatin remodelling complexes in heart development. Nature. 2004; 432:107-112. [PubMed: 15525990] 

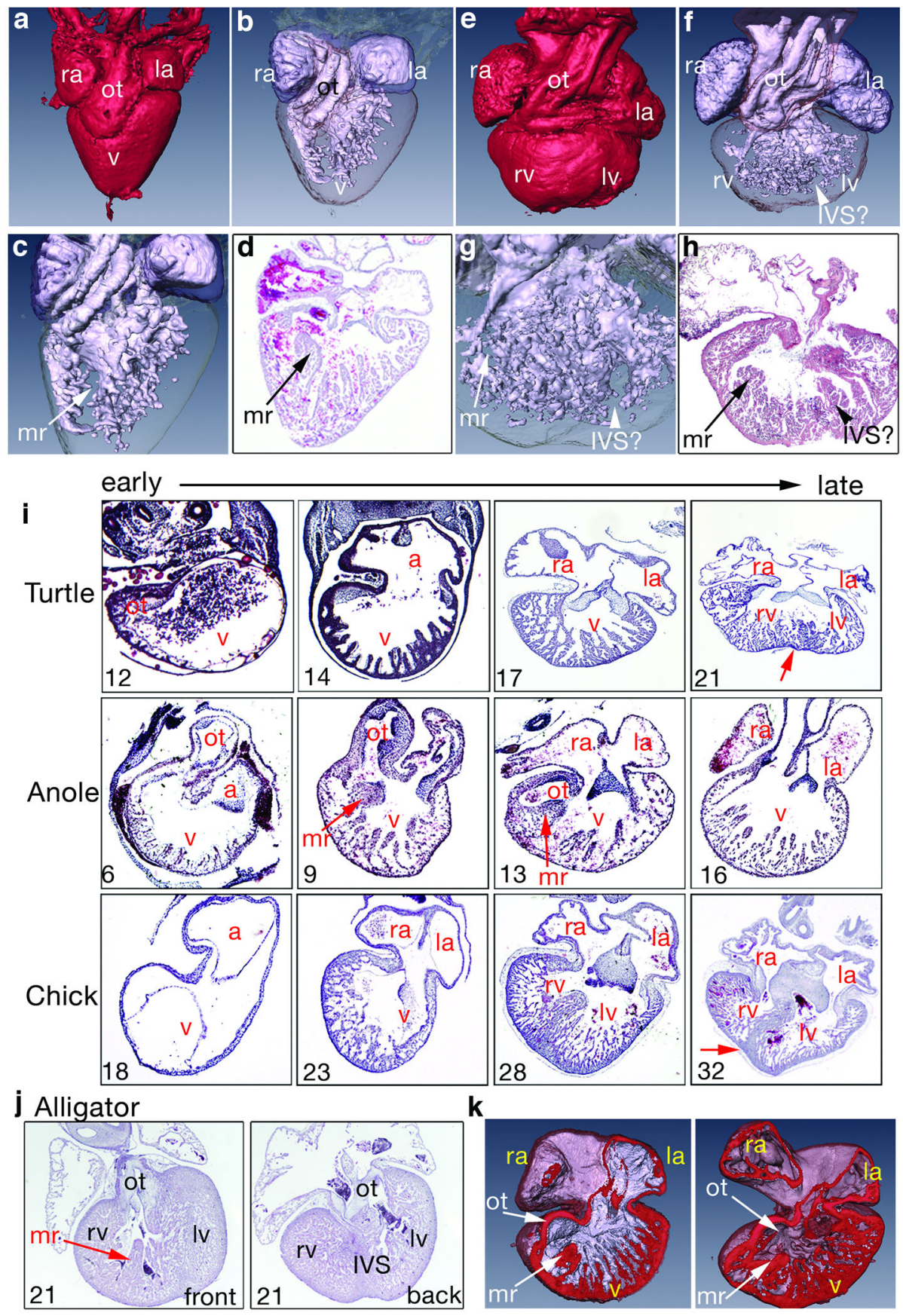

Figure 1. Reptilian heart development

$\mathbf{a}-\mathbf{h}$. Optical projection tomography of post-hatching anole (a-d) and turtle (e-h) hearts. a,e external view, b,c,f,g chamber fill; $\mathbf{d}, \mathbf{h}$ histology. i, Histological analysis of heart development in turtle, anole, and chick embryos. Four representative stages shown are equivalent between species. arrow: interventricular groove. j. Histology of stage 21 embryonic alligator heart. k. OPT of st. 17 (left) and st. 21 (right) turtle hearts. In all reptile embryos (i-k), note close apposition of mr and ot. a: atrium, IVS?: IVS-like structure, la: 
left atrium, lv: left ventricle, mr: muscular ridge, ot: outflow tract, ra: right atrium, rv: right ventricle. 


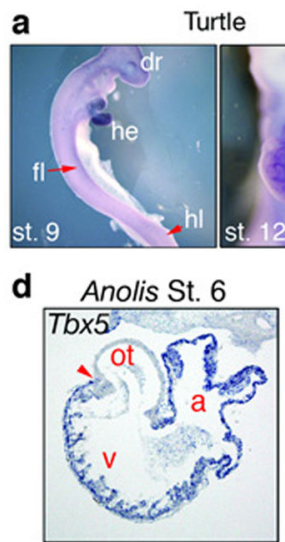

Chick St. 20

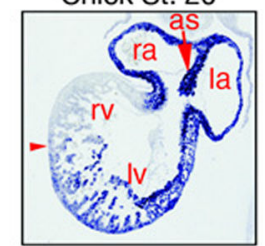

g
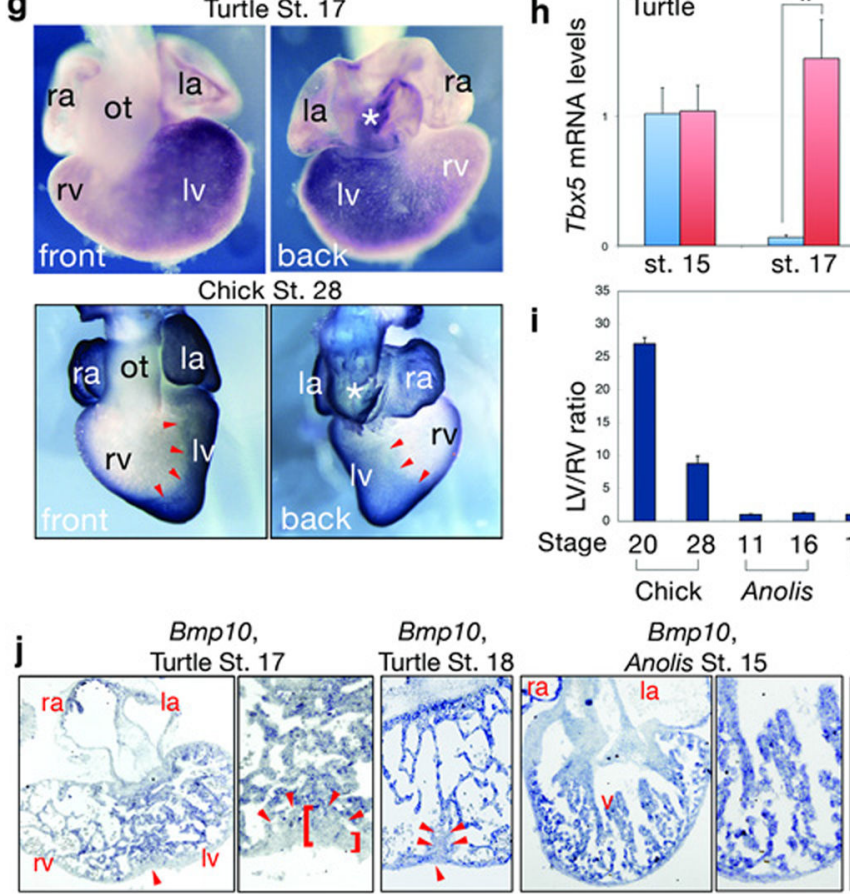

C Mouse

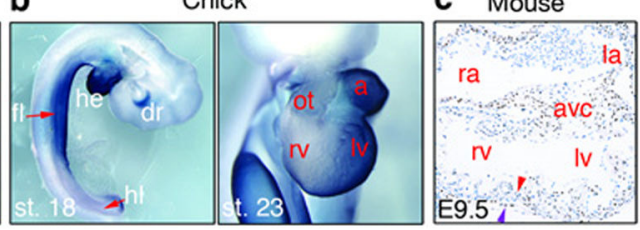

e Anolis St. $13 \quad$ Turtle St. $15 \quad$ Chick St. 28



f Anolis St. $16 \quad$ Turtle St. 17 Chick St. 30
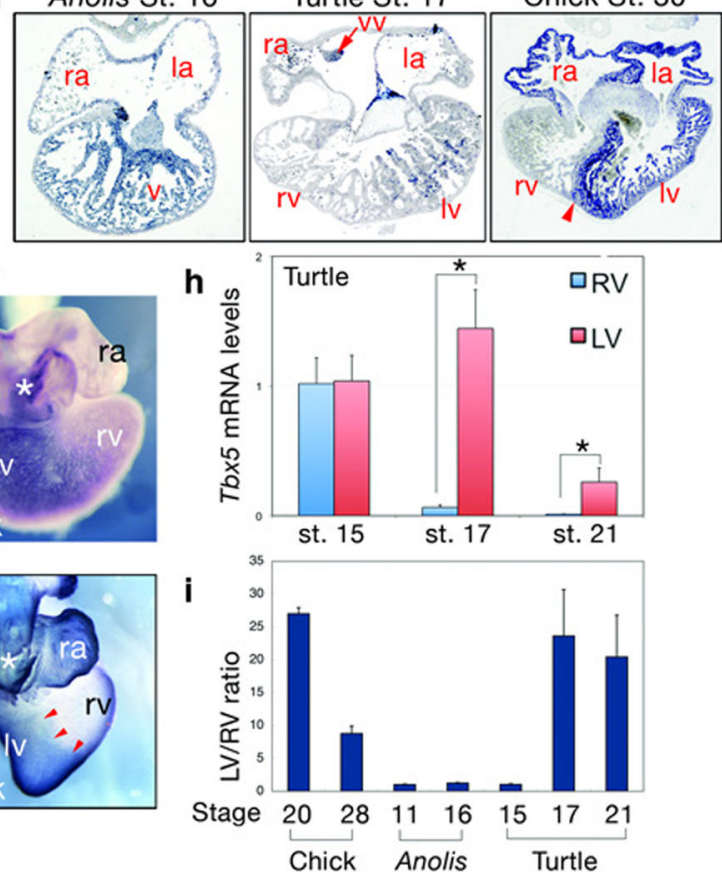

Figure 2. Gene expression in amniote embryos

a-c, Tbx5 expression in turtle, chick, and mouse. Left panels: whole-embryo views. He: heart, fl: forelimbs, dr: dorsal retina, hl: hindlimbs. Right panels: closeup ventral views of embryonic hearts. In c, bottom panel shows Tbx5 immunohistochemistry; red arrowheads: rv/lv junction; purple arrowhead: epicardium. la: left atrium, lv:left ventricle, ot: outflow tract, ra: right atrium, rv: right ventricle. d-g, Expression of Tbx5. As: atrial septum; avc: atrioventricular cushion. Arrowheads mark the boundary between lv and rv, or V and OT for Anole in d. h. Quantitation of Tbx5 mRNA levels in turtle LV and RV; data are mean \pm SD 
normalized to St $15 \mathrm{RV} .{ }^{*} P<0.005$ by t-test. i. Ratio of $T b x 5$ mRNA levels between the LV and RV. j. Bmp10 expression in turtle and Anole hearts. Arrowheads: interventricular groove and septum. Brackets: thickness of Bmp10-negative area. k. Nppa expression in the turtle is in a left-right gradient similar to $T b x 5$. 

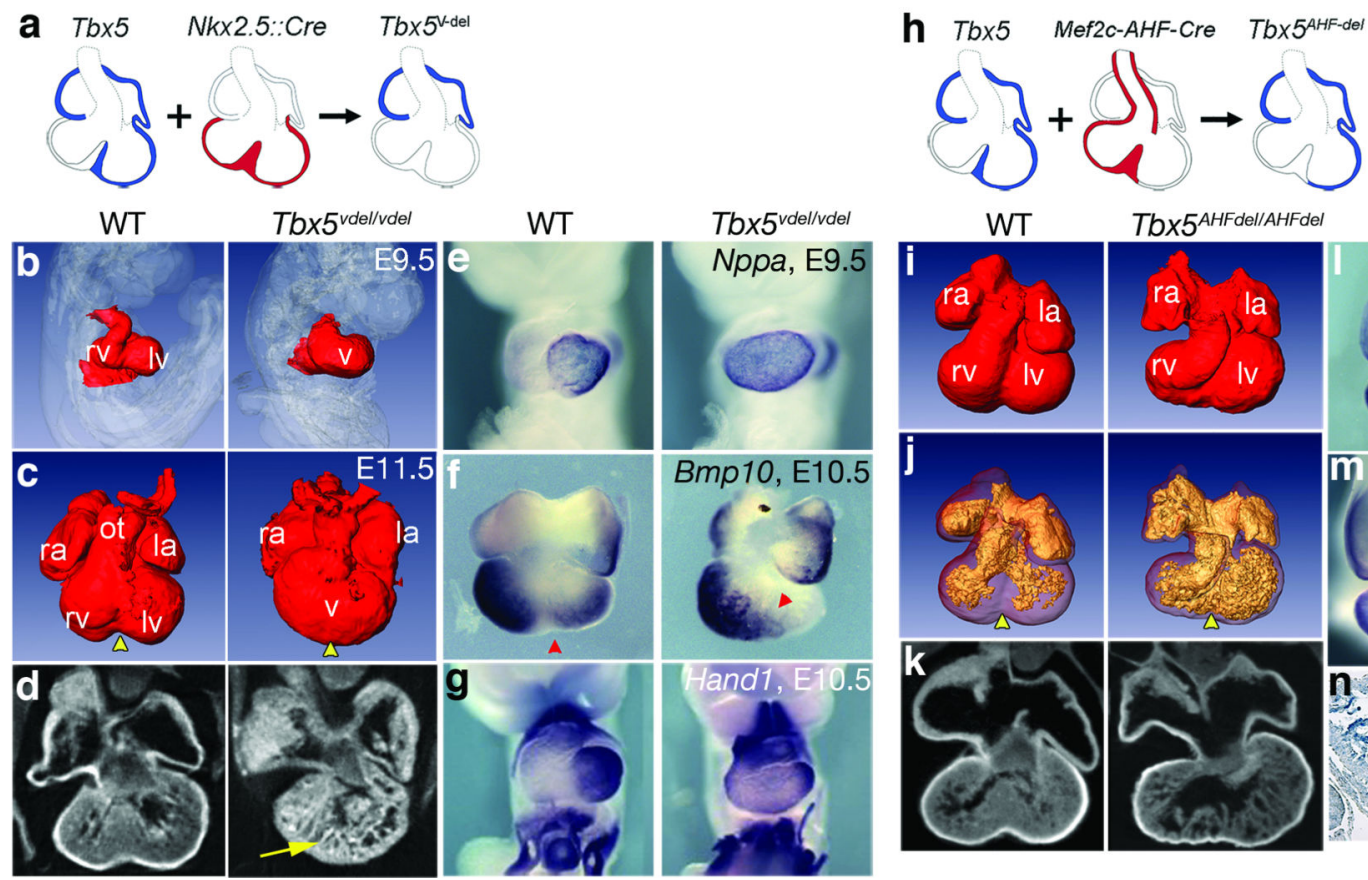

$T b \times 5^{A H F d e l / A H F d e /}$

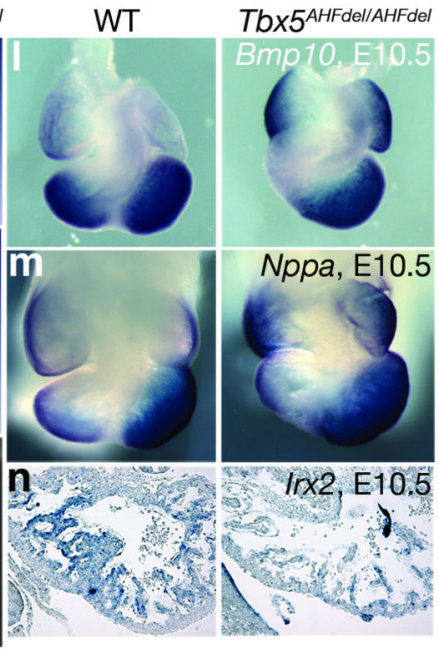

Figure 3. Ventricle-restricted deletion of mouse Tbx5

a, Strategy for ventricular deletion of Tbx5. b-d, OPT of wild-type (WT) and Tbx $5^{v d e l / v d e l}$ embryos and hearts at E9.5 (b) and E11.5 (c, d). Arrowheads indicate position of the IVS. e,f,g: Gene expression for indicated transcripts. h, Strategy for Tbx5 deletion in anterior heart field derivatives. I-k, OPT of wild-type (WT) and Tbx $5^{\text {AHFdel/AHFdel }}$ hearts at E10.5. i: external view, j: chamber fill, k: virtual sections. l,m,n: Gene expression for indicated transcripts. 

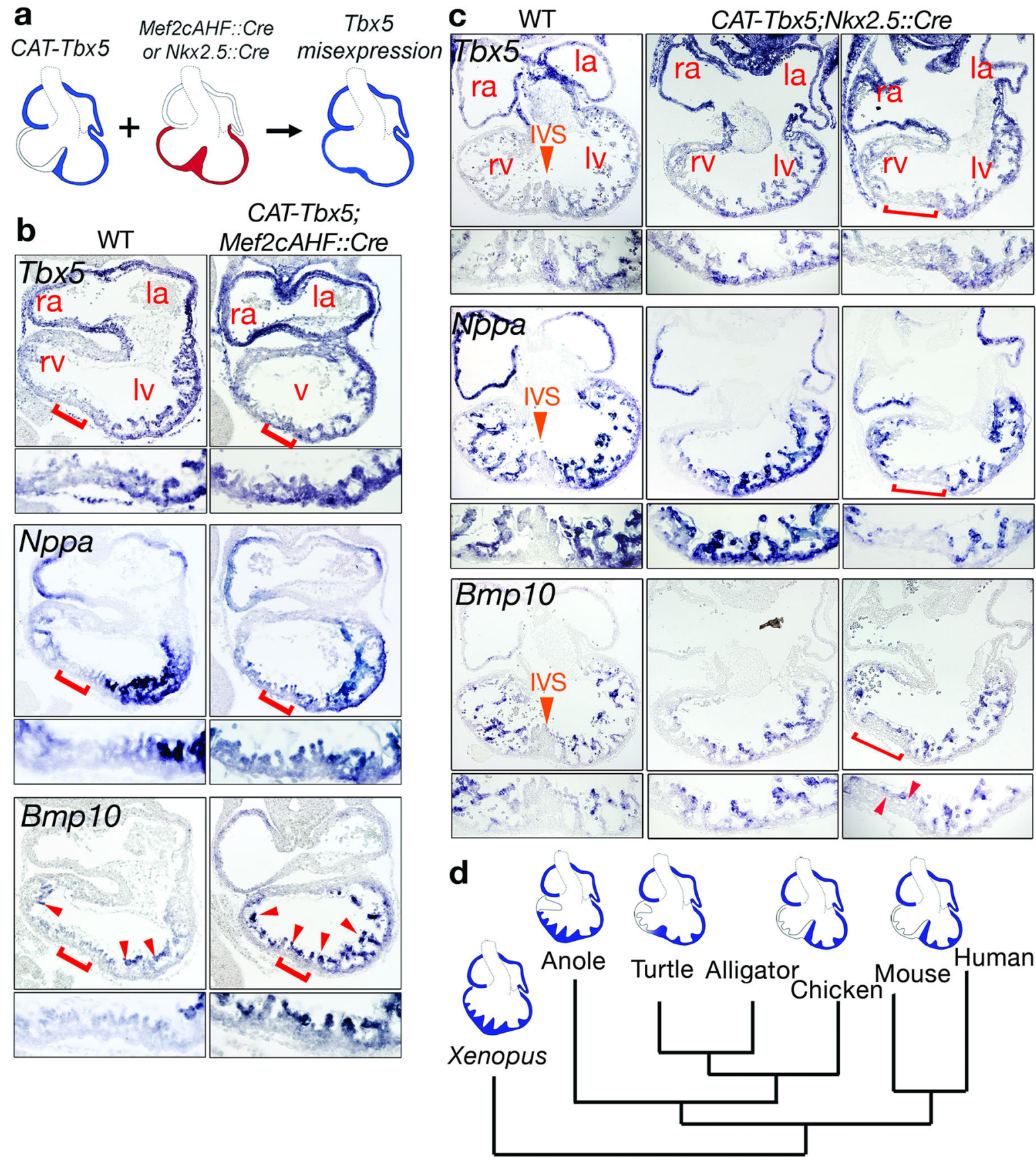

Figure 4. Misexpression of $T b x 5$ results in loss of IVS patterning

a. Strategy for ventricular misexpression of $T b x 5$. b. Morphology and gene expression in CAT-Tbx5;Mef2cAHF::Cre embryos for indicated transcripts. Brackets: IVS region, magnified in lower panels. Arrowheads: trabecular Bmp10 expression. c. Morphology and gene expression in CAT-Tbx5;Nkx2.5::Cre embryos at E11.5. Orange arrows: interventricular septum region (IVS). Brackets show a rudimentary septum in a mutant embryo. d. Diagrammatic representation of embryonic heart structures and patterns of $T b x 5$ 
expression (blue) in vertebrate evolution. la: left atrium, lv: left ventricle, ot: outflow tract, ra: right atrium, rv: ventricle, v: ventricle. 